\title{
Cryoplasty Versus Conventional Balloon Angioplasty of the Femoropopliteal Artery in Diabetic Patients: Long-Term Results from a Prospective Randomized Single-Center Controlled Trial
}

\author{
Stavros Spiliopoulos • Konstantinos Katsanos • \\ Dimitris Karnabatidis • Athanasios Diamantopoulos • \\ George C. Kagadis $\cdot$ Nikolaos Christeas $\cdot$ Dimitris Siablis
}

Received: 9 March 2010/Accepted: 28 May 2010/Published online: 24 June 2010

(C) Springer Science+Business Media, LLC and the Cardiovascular and Interventional Radiological Society of Europe (CIRSE) 2010

\begin{abstract}
The purpose of this study was to investigate the immediate and long-term results of cryoplasty versus conventional balloon angioplasty in the femoropopliteal artery of diabetic patients. Fifty diabetic patients (41 men, mean age 68 years) were randomized to cryoplasty (group CRYO; 24 patients with 31 lesions) or conventional balloon angioplasty (group COBA; 26 patients with 34 lesions) of the femoropopliteal artery. Technical success was defined as $<30 \%$ residual stenosis without any adjunctive stenting. Primary end points included technical success, primary patency, binary in-lesion restenosis $(>50 \%)$, and freedom from target lesion recanalization. Cox proportional hazards regression analysis was performed to adjust for confounding factors of heterogeneity. In total, $61.3 \%$ (19 of 31) in group CRYO and $52.9 \%$ (18 of 34) in group COBA were de novo lesions. More than $70 \%$ of the lesions were Transatlantic Inter-Society Consensus (TASC) B and C in both groups, and $41.4 \%$ of the patients in group CRYO and $38.7 \%$ in group COBA suffered from critical limb ischemia. Immediate technical success rate was $58.0 \%$ in group CRYO versus $64.0 \%$ in group COBA $(p=0.29)$. According to 3 -year Kaplan-Meier estimates, there were no significant differences with regard to patient survival (86.8\% in group CRYO vs. $87.0 \%$ in group COBA, $p=0.54$ ) and limb salvage ( 95.8 vs. $92.1 \%$ in groups CRYO and COBA, respectively,
\end{abstract}

S. Spiliopoulos $(\bowtie) \cdot$ K. Katsanos · D. Karnabatidis .

A. Diamantopoulos · N. Christeas · D. Siablis

Department of Diagnostic and Interventional Radiology,

Angiography Suite, School of Medicine, Patras University

Hospital, Patras, Rion 26504, Greece

e-mail: stavspiliop@upatras.gr

G. C. Kagadis

Department of Medical Physics, School of Medicine, Patras

University Hospital, Patras, Rion 26504, Greece $p=0.60$ ). There was a nonsignificant trend of increased binary restenosis in group CRYO (hazard ratio [HR] 1.3; $95 \%$ CI $0.6-2.6, p=0.45$ ). Primary patency was significantly lower in group CRYO compared with group COBA (HR 2.2; $95 \%$ CI $1.1-4.3, p=0.02$ ). Significantly more repeat intervention events because of recurrent symptoms were required in group CRYO (HR 2.5; 95\% CI 1.2-5.3, $p=0.01$ ). Cryoplasty was associated with lower primary patency and more clinically driven repeat procedures after long-term follow-up compared with conventional balloon angioplasty.

Keywords Cryoplasty - Balloon angioplasty · Femoropopliteal artery $\cdot$ Diabetes

\section{Introduction}

Diabetes mellitus (DM) is developing into a worldwide epidemic with an estimated prevalence of $5.4 \%$, and $>300$ million people will be affected by the year 2025 [1]. DM is a well-recognized independent risk factor of atherosclerosis and peripheral arterial disease, inducing multilevel, mainly small-vessel disease of the coronary and infrapopliteal arteries [1,2]. Owing to a higher prevalence of diabetic foot ulcers and critical limb ischemia (CLI), diabetic patients demonstrate a 5 times higher risk of amputation and a 10 times higher risk of mortality after percutaneous treatment of peripheral arterial occlusive disease (PAOD) compared with normoglycemic patients [3]. Of note, almost half of all amputees suffer from DM [4]. Interestingly, DM has been identified as an independent risk factor of recurrent occlusive disease symptoms, and there is a trend toward decreased vascular patency and higher rates of repeat intervention procedures after percutaneous or surgical revascularization of the infrainguinal arteries [5-9]. 
Despite their safety and versatility, current results of minimally invasive endovascular techniques for atherosclerotic lesions in the superficial femoral (SFA) and popliteal (PA) arteries remain mediocre, with reported 2year patency rates between 40 and $60 \%$ [10, 11]. Low primary patency and increased repeat intervention rates, mainly due to vascular restenosis by means of intimal hyperplasia and negative vessel wall remodelling, has orientated modern cardiovascular research toward new alternative techniques, such as atherectomy, laser-assisted angioplasty, covered stent-grafts, brachytherapy, and cryoplasty [12-14]. Of note, there is no controlled evidence in the literature regarding percutaneous therapies of the femoropopliteal artery in the setting of DM apart from single-center patient cohorts [15]. Especially with regard to new minimally invasive technologies, such as cryoplasty, no properly designed, randomized controlled trials are available [16, 17]. We conducted a prospective, singlecenter, randomized, controlled trial comparing cryoplasty with conventional balloon angioplasty for the treatment of femoropopliteal arterial occlusive disease in the demanding population of DM patients. The investigators report their immediate and long-term clinical and angiographic results.

\section{Materials and Methods}

\section{Study Design}

Institutional Review Board approval was obtained by the Hospital's Ethical and Scientific Committee. The nature of the study was explained to all potential subjects, including the fact that there would be an equal possibility that a patient could be assigned to either study arm. Patients were further informed that this was designed to be a long-term study and signed the appropriate informed consent form. The randomization was performed with pooled 1:1 envelopes, which were opened only after lesions were successfully crossed.

Study inclusion and exclusion criteria are listed in Table 1 . The primary patient inclusion criteria were noninsulin (NIDDM)- or insulin-dependent DM (IDDM) and atherosclerotic peripheral arterial disease with symptoms of severe claudication or CLI (Rutherford stages 3 to 6). Hemodynamically significant stenosis $(\geq 70 \%$ by visual estimation) or occlusion of the SFA and/or the PA, as documented by either digital subtraction angiography (DSA) or computed tomography angiography, was a mandatory angiographic inclusion criterion. Both de novo and in-stent restenotic (ISR) lesions were enrolled. Patients with diet-controlled diabetes, a history of severe contrast allergy or hypersensitivity, intolerance to aspirin and/or clopidogrel, systemic coagulopathy or hypercoagulation disorders, acute limb ischemia, Buerger disease, deep vein
Table 1 Inclusion and exclusion criteria

Inclusion criteria
NIDDM or IDDM
Severe claudication or CLI (Rutherford stage 3 to 6)
Stenosis $\geq 70 \%$ or occlusion of the SFA and/or the PA
De novo and ISR lesions
Exclusion criteria
Diet-controlled diabetes
History of severe contrast allergy or hypersensitivity
Intolerance to aspirin and/or clopidogrel
Systemic coagulopathy or hypercoagulation disorders
Acute limb ischemia
Buerger disease
Deep vein thrombosis
Infected tissue loss
Absent pedal arch runoff

$S F A$ superficial femoral artery, $P A$ popliteal artery

thrombosis, infected tissue loss, and absent pedal arch runoff were excluded from the trial.

Procedure

All patients were premedicated with aspirin $(100 \mathrm{mg} / \mathrm{d})$ and clopidogrel $(75 \mathrm{mg} / \mathrm{d})$ for at least 3 days before the procedure. Patients who were receiving metformin for their diabetes were adequately hydrated and properly advised to temporarily modify their therapy as determined by European Society of Urogenital Radiology (ESUR) guidelines [18].

An antegrade or retrograde femoral artery access using an appropriate-sixe sheath (6F to $7 \mathrm{~F}$ ) was performed. A bolus dose of unfractionated heparin (3000 to $5000 \mathrm{IU}$ ) was administered immediately after sheath placement, and an infusion rate of $1000 \mathrm{U} / \mathrm{h}$ was maintained during the rest of the procedure. Routine endovascular maneuvers using standard guidewires and catheters were used to cross the SFA and/or the PA lesion as needed. Lesion treatment was then randomized either to (1) cryoplasty with the PolarCath balloon (PolarCath Peripheral Dilatation System; Boston Scientific, Natick, MA) or (2) conventional balloon angioplasty with commercially available semicompliant or noncompliant balloon catheters (inflation period 60 to 120 s). In all cases, balloon size was chosen according to reference vessel diameter per visual estimate. Balloon length was chosen to match lesion length, and if that was not possible, to slightly exceed it, according to routine clinical practice. Stenting was reserved for bail-out in case of elastic recoil, postdilatation residual stenosis $>30 \%$, or severe flow-limiting dissection (type C). Outflow obstructive lesions of the infrapopliteal arteries were treated as 
necessary to improve runoff. At the end of the procedure, hemostasis was achieved with manual compression or a closure device. Patients were routinely hospitalized overnight and discharged the next day with a prescription for dual antiplatelet therapy $(75 \mathrm{mg} / \mathrm{d}$ clopidogrel and $100 \mathrm{mg} /$ $\mathrm{d}$ aspirin) for 6 weeks and solely clopidogrel or aspirin indefinitely thereafter.

\section{Cryoplasty Treatment}

Cryoplasty therapy was performed with the use of the PolarCath Peripheral Dilatation System, which includes an over-the-wire, double-lumen dilatation balloon catheter manufactured of Pebax ${ }^{\circledR}$ (Atochem Inc, PA, USA) and an inflation system consisting of a microprocessor unit and a nitrous oxide cartridge. The cryoplasty catheter is formed by three layers (inner, middle, and outer), and its fluoroscopic visibility is attained by radiopaque markers placed in the middle layer.

Balloon inflation is achieved by a specially designed apparatus that releases liquid nitrous oxide from the specially designed high-pressure cartridge through the catheter lumen and into the lower-pressure balloon chamber, where it changes state from liquid to gas and expands its volume. The whole phenomenon is based on the Thomson-Joule effect $(\mu=\Delta T / \Delta P)$. The rapid expansion of the gas volume inflates the balloon and produces a rapid decrease in temperature, thus effectively cooling the balloon surface and the adjacent vessel wall to $10^{\circ} \mathrm{C}$ while the inflation pressure gradually reaches $8 \mathrm{Atm}$, the pressure of which is tightly regulated by a valve mechanism attached to the double-balloon catheter. The inflation-active cooling/ deflation-passive thawing time period ranges from 26 to $30 \mathrm{~s}$. The technique theoretically aims at both better immediate and long-term results because cooling of the atherosclerotic plaque and its elastic components is believed to achieve a more uniform and less traumatic angioplasty, resulting in better plaque remodelling with less flow-limiting dissections and vessel wall recoiling. Several in vitro studies have shown that the specific circle of $-10^{\circ} \mathrm{C}$ active cooling/passive thawing creates a dehydration/rehydration effect in the adjacent cells through the a change in the osmotic forces acting in the vessel area around the catheter. The whole cryoplasty procedure is believed to induce cell apoptosis (programmed cell death enhancement) rather than necrosis of the remaining endothelium and of the smooth muscle cells of the middle vessel layer and may therefore result in less inflammation, less neointimal hyperplasia, and consequently decreased vascular restenosis [19-21]. Because the time span and pressure limit of the cryoplasty catheter cannot be adjusted by the operator, a second inflation session with the same PolarCath balloon, as deemed necessary, was performed in cases of tight resistant lesions to achieve a satisfactory final result.

Follow-Up, End Points, and Definitions

Patients were prospectively scheduled for regular clinical and angiographic follow-up visits at 6 months, 1 year, and annually thereafter unless clinical deterioration indicated differently. Patient mobility status was interrogated, and all angiographic end points were evaluated with intra-arterial DSA.

Primary end points included technical success, primary patency, binary in-lesion restenosis, and freedom from target lesion recanalization (TLR). Secondary clinical end points included 30-day complications, patient mortality, limb salvage, and minor amputation of the treated limbs. Technical success for group CRYO was defined as a successful angioplasty result with residual lesion stenosis $<30 \%$ on visual estimate and without adjunctive use of an additional high-pressure balloon or bail-out stent. In group COBA, technical success was defined as a successful angioplasty result with residual lesion stenosis $<30 \%$ on visual estimate and without bail-out stenting. Lesions requiring additional angioplasty with a conventional balloon because of a suboptimal postangioplasty result, even after a maximum of two cryoplasty sessions, were classified as cryoplasty technical failures.

Primary patency was defined as angiographic visualization of a nonoccluded lesion and no need for any additional repeat interventional procedure within the previously treated lesion. Absent or thread-like blood flow were classified as vascular occlusions. Binary in-lesion restenosis was defined as vascular restenosis of the treated lesion $>50 \%$ threshold according to reference vessel diameter. TLR included any additional recanalization procedure within the area of the treated femoropopliteal lesion because of clinical deterioration and relapse of symptoms (i.e., clinically driven repeat procedures). The rest of the secondary clinical end points were defined according to published international guidelines and reporting standards [22].

SFA lesions were initially classified according to Transatlantic Inter-Society Consensus (TASC) guidelines. However, during data analysis of the study they were retrospectively modified according to the more recent TASC II guidelines [23]. Infrapopliteal runoff arteries were counted and graded with a simplified scoring system ( 0 to 2) as described elsewhere [24]. Baseline and follow-up angiographic images were reviewed by two independent expert vascular interventional radiologists using the dedicated computerized vessel analysis software of the angiography unit. A consensus was reached in case of borderline differences. 
Statistical Analysis

Discrete variables were expressed as counts and percentages, and continuous variables were given as medians and interquartile ranges (IQR: i.e., between the 25 th and 75 th percentiles) in parentheses or as means \pm SE if they passed the normality test as stated later in the text. KolmogorovSmirnov goodness-of-fit test was used to determine whether continuous data should be treated as originating from normal distributions. Unpaired Student $t$ test was used to test the significance of difference of variables that passed the normality test. Mann-Whitney test was used for qualitative variables and for nonparametric testing of continuous variables that did not pass the normality test. Comparison of two proportions was done by testing the null hypothesis that the proportions were equal, with an appropriate quantity as a standardized normal deviate test. Life-table analysis with the Kaplan-Meier method was employed for calculation of the cumulative proportion outcomes up to 36-month angiographic and 42-month clinical follow-up time-points. Kaplan-Meier curves were compared with the log-rank test.

Results were stratified according to type of treatment (cryoplasty vs. COBA). To identify independent risk factors affecting the analyzed end points, stepwise regression analysis was performed using the Cox proportional-hazards regression model during the total follow-up period. Dependent variables were IDDM, increased serum creatinine level $(>1.5 \mathrm{mg} / \mathrm{dL})$, nicotine use ( $\leq 12$ months), hyperlipidemia (more than mild increase) controlled with diet or drugs), initial lesion grade (stenosis or occlusion), TASC II lesion classification, lesion type (de novo or ISR), runoff score (0 to 3), heavy calcifications of the lesion, and treatment modality (cryoplasty vs. COBA). The covariates included primary patency, binary in-lesion restenosis, and TLR-free survival. The results were expressed as HRs with 95\% confidence intervals (CIs) and the associated level of statistical significance. In cases of significant results, the adjusted curve plots of the identified covariate are presented. Statistical analysis was performed using the SPSS/ PASW statistical software package (version 17.0; SPSS/ PASW, Chicago, IL).The threshold of statistical significance was set at $p=0.05$.

\section{Results}

Between January 2005 and October 2007, a total of 50 patients (41 men and 9 women) with a mean age of $68 \pm$ 9.5 years (range 47 to 82 ), who were scheduled for percutaneous recanalization of the SFA or the PA due to PAOD, gave informed consent and were randomly assigned to one of the two groups. Twenty-four patients
(29 limbs with 31 lesions) were treated with cryoplasty (group CRYO), whereas the remaining 26 patients (31 limbs with 34 lesions) underwent conventional balloon angioplasty (group COBA) and served as the control group. The followup completion date was May 2009. Various balloon types commercially available in our department, including semicompliant and noncompliant high-pressure balloons, were applied in the COBA control group as follows: (1) Ultra-Thin Diamond and Blue Max PTA; Boston Scientific, Natick, MA; (2) Profiler; Angiodynamics, Latham, NY; and (3) Cronus; Medispes WS AG, Zug, Switzerland.

Baseline demographics, anatomic lesion characteristics, and procedural variables of both groups are listed in Tables 2 and 3. The majority of the patients treated had severe intermittent claudication classified as Rutherford stage 3 (58.6\% in group CRYO vs. $61.3 \%$ in group COBA, $p=0.42$ ). There was an almost equal distribution of de novo and ISR lesions in both groups, but there were no

Table 2 Baseline patient demographics and clinical status

\begin{tabular}{llll}
\hline Demographics & Cryoplasty & COBA & $p$ \\
\hline Patients $(n)$ & 24 & 26 & - \\
Limbs $(n)$ & 29 & 31 & - \\
Male sex & $21(87.5)$ & $22(84.6)$ & 0.38 \\
Age (y) & $65.3 \pm 10.4$ & $70.3 \pm 7.8$ & 0.12 \\
Smoking habit & $12(50)$ & $11(42.3)$ & 0.29 \\
IDDM & $10(41.7)$ & $9(34.6)$ & 0.30 \\
Hyperlipidemia & $17(71.0)$ & $15(58.0)$ & 0.16 \\
Arterial hypertension & $23(95.8)$ & $23(88.5)$ & 0.16 \\
Carotid disease & $9(37.5)$ & $5(19.2)$ & 0.07 \\
Cardiac disease & $7(29.2)$ & $10(38.5)$ & 0.35 \\
Renal disease & $8(33.3)$ & $4(15.4)$ & 0.06 \\
Physical health status & & & \\
ASA 1 to 2 & $18(75.0)$ & $20(77.0)$ & 0.43 \\
ASA 3 & $6(25.0)$ & $6(23.0)$ & 0.43 \\
Rutherford category of PAD & & \\
Stage 3 & $17(58.6)$ & $19(61.3)$ & 0.42 \\
Stage 4 & $10(34.5)$ & $6(19.4)$ & 0.09 \\
Stage 5 & $1(3.4)$ & $4(12.9)$ & 0.09 \\
Stage 6 & $1(3.4)$ & $2(6.5)$ & 0.29 \\
CLI & $12(41.4)$ & $12(38.7)$ & 0.41 \\
\hline Contin & & & \\
\hline
\end{tabular}

Continuous data are presented as mean $\pm \mathrm{SE}$; categoric data are given as counts and percentages in the parentheses

ASA American Society of Anesthesiologists

a Transient ischemic attack or stroke

b History of myocardial infarction (MI) or occult MI by electrocardiogram, stable or unstable angina, controlled arrythmia, drug-compensated congestive heart failure (CHF), poorly controlled arrhythmia, and poorly compensated CHF

c ASA class 1 denotes a healthy individual; class 2 denotes mild systemic disease; and class 3 denotes severe but not incapacitating disease 
Table 3 Procedural variables

\begin{tabular}{llll}
\hline Variables & Cryoplasty & COBA & $p$ \\
\hline Lesions treated $(n)$ & 31 & 34 & - \\
TASC A & $4(13.8)$ & $6(19.4)$ & 0.2 \\
TASC B & $17(58.6)$ & $12(38.7)$ & 0.06 \\
TASC C & $8(27.6)$ & $11(35.5)$ & 0.02 \\
TASC D & $0(0.0)$ & $2(6.5)$ & 0.08 \\
De novo lesions & $19(61.3)$ & $18(52.9)$ & 0.2 \\
ISR lesions & $12(38.7)$ & $16(47.1)$ & 0.2 \\
Heavy calcifications & $9(29.0)$ & $10(29.4)$ & 0.4 \\
Initial occlusions & $7(22.6)$ & $5(14.7)$ & 0.2 \\
Mean treated lesion length $(\mathrm{cm})^{\mathrm{a}}$ & $11.9 \pm 5.1$ & $12 \pm 6.0$ & 0.91 \\
Mean stented lesion length $(\mathrm{cm})^{\mathrm{a}}$ & $50 \pm 18.5$ & $68.3 \pm 34.3$ & 0.28 \\
Mean balloon diameter $(\mathrm{mm})$ & $5.7 \pm 0.65$ & $5.7 \pm 0.57$ & 0.5 \\
Subintimal approach & $2(6.5)$ & $2(5.9)$ & 0.4 \\
Popliteal artery & $4(12.9)$ & $2(5.9)$ & 0.16 \\
\hline
\end{tabular}

Categoric data are given as counts and percentages in parentheses

${ }^{a}$ Data are presented as mean $\pm \mathrm{SD}$

significant differences (Table 3). Most of the lesions treated were characterized as TASC B and C lesions according to the revised classification system $(86.2 \%$ in group CRYO vs. $74.2 \%$ in group COBA, $p=0.21$ ). The average lesion length was $11.9 \pm 5 \mathrm{~cm}$ in group CRYO and $12.0 \pm 6 \mathrm{~cm}$ in group COBA $(p>0.05)$. Almost $29 \%$ of the lesions in both groups were characterized as heavily calcified, whereas a similar number of initial occlusions were treated (22.6 vs. $14.7 \%$ in groups CRYO and COBA, respectively, $p=0.22)$. Only 4 lesions were treated using the subintimal technique $(6.5 \%$ in group $\mathrm{A}$ and $5.9 \%$ in group $\mathrm{B}, p=$ 0.46). The minority of the lesions (4 of 31 lesions $[12.8 \%$ in group A] and 2 of 34 lesions [5.9\% in group B], $p=$ 0.16) were located in the PA. At the end of the procedure all treated limbs had at least one runoff vessel to the distal foot.

Technical success rate was similar between the two groups $(58.0 \%$ in group CRYO vs. $64.0 \%$ in group COBA, $p=0.29$ ). Our protocol included a balloon-inflation time of 60 to 120 seconds in group COBA, and the duration of an eventual second inflation was 120 seconds (mean time 82.9 \pm 29.5 ). In group CRYO, more than half of the lesions $(64.5 \%)$ required a second cryoplasty application due to suboptimal angioplasty result or flow-limiting dissection. In nine cases $(29.0 \%)$, the additional aid of a conventional angioplasty balloon was required to achieve immediate technical success. The incidence of flow-limiting (type C) dissections was analogous in both arms (16.1\% in group CRYO vs. $17.6 \%$ in group COBA, $p=0.435)$. Regarding the overall need for stenting, there was a trend toward less stent use for cases in which cryoplasty treatment was used as initial treatment (25.8 vs. $35.3 \%$ in group COBA, $p=0.2$ ). In addition, overall stent length was less in the cryoplasty group, but this was not statistically significant (mean stent length $50 \mathrm{~mm}$ in group CRYO vs. $68 \mathrm{~mm}$ in group COBA, $p=0.28$ ). No immediate procedure-related complications (thromboembolic events, hemorrhage, or death) occurred. The final semiquantitative runoff score was significantly higher in group CRYO (runoff score 2 [2] vs. $1[1,2]$ in group COBA, $p=0.002$ ). Immediate procedural outcomes are listed in Table 4.

Angiographic and clinical follow-up periods were similar in both patient arms: The mean angiographic follow-up period was $23.5 \pm 1.9$ months in group CRYO vs. $25.3 \pm$ 2.0 months in group COBA $(p=0.6)$, whereas the mean clinical follow-up period was $32 \pm 9$ months in group CRYO vs. $32 \pm 2$ months in group COBA, respectively ( $p$ $=0.7$, without any significant differences in patient compliance between the two groups. Only one patient ( 1 of $24 ; 4.16 \%$ ) assigned to group CRYO was lost from angiographic but not from clinical follow-up after 6 months due to an ischemic stroke. After 3 years, there were no significant differences with regard to patient survival $(86.8 \%$ in group CRYO vs. $87.0 \%$ in group COBA; $p=0.54$ by log-rank test) and limb salvage (95.8 vs. $92.1 \%$ in groups CRYO and COBA, respectively; $p=0.60$ by logrank test), according to life-table survival analysis (Fig. 1). None of the deaths were related to the procedure. Minor amputation rates were similar in the two study arms $(6.9 \%$ in group CRYO vs. $9.7 \%$ in group COBA, $p=0.3$ ).

According to Kaplan-Meier estimates from bivariable survival analysis, angiographic primary patency $\leq 36$ months was not significantly different between the two groups (66.6 vs. $67.6 \%, 59.2$ vs. $54.6 \%$, and 59.2 vs. $54.6 \%$ in

Table 4 Procedural outcomes

\begin{tabular}{llll}
\hline Outcomes & Cryoplasty & COBA & $p$ \\
\hline Technical success & $18(58.0)$ & $22(64.7)$ & 0.4 \\
Flow-limiting dissection (type C) $^{5(16.1)}$ & $6(17.6)$ & 0.2 \\
Adjunctive stent use $^{\mathrm{a}}$ & $8(25.8)$ & $12(35.3)$ & 0.2 \\
Final runoff score $^{\mathrm{b}}$ & $2(2-2)$ & $1(1-2)$ & 0.002 \\
Puncture-site related complications & & \\
$\quad$ Major & $0(0.0)$ & $0(0.0)$ & - \\
$\quad$ Minor & $1(3.5)$ & $1(3.2)$ & 0.4 \\
Procedure related adverse events & $0(0.0)$ & $0(0.0)$ & - \\
Minor amputations & $2(6.9)$ & $3(9.7)$ & 0.3 \\
Double application of cryoplasty & $20(64.5)$ & - & - \\
COBA mean inflation time (s) & - & $82.9 \pm 29.5$ & - \\
\hline
\end{tabular}

Categoric data are given as counts with percentages in parentheses

${ }^{\text {a }}$ Number of cases in which stents were placed due to flow limiting dissection and/or elastic recoil

${ }^{\mathrm{b}}$ Median with IQR in parentheses

${ }^{c}$ Data presented as mean $\pm \mathrm{SE}$ 
Fig. 1 A Patient survival and B limb salvage Kaplan-Meier analysis for groups CRYO and COBA
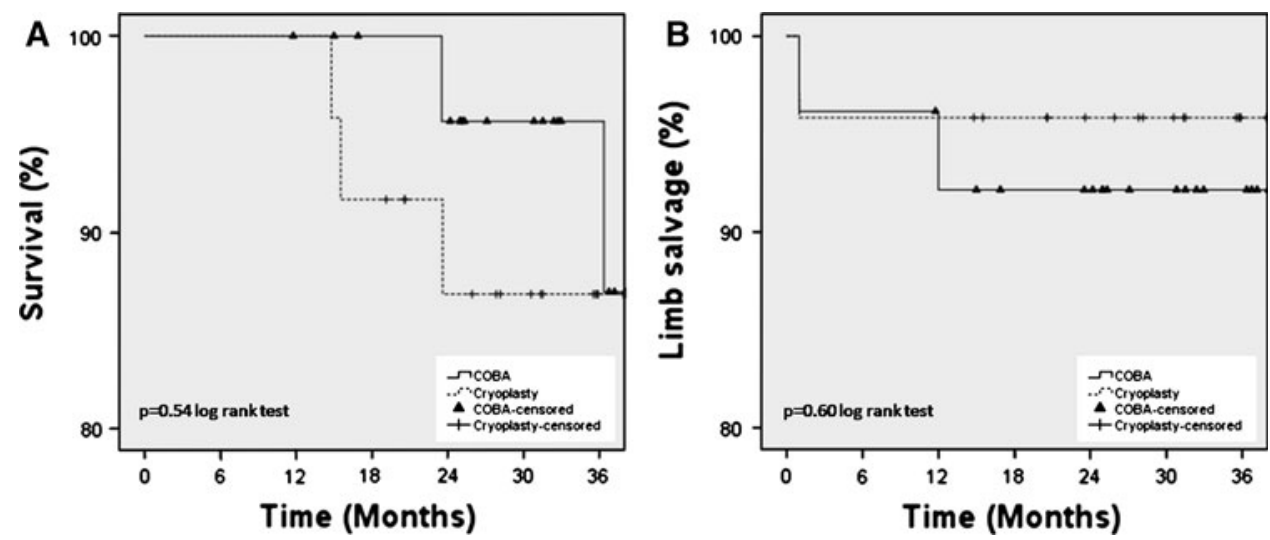

group CRYO vs. group COBA at 12,24 , and 36 months respectively, $p=0.894$ by log-rank test). Numbers of lesions at risk in group CRYO were 12, 7, and 2 and in group COBA were 22,12 , and 4 at 12, 24 and 36 months, respectively. However, after adjustment for confounding factors of heterogeneity with multivariable Cox model, cryoplasty was identified as a strong independent predictor for decreased primary patency (HR 2.2, 95\% CI 1.1-4.3, $p=0.02$; Table 5 and Fig. 2). De novo atheromatous lesions were also identified as a strong independent predictor of decreased inlesion primary patency compared with ISR lesions (HR 2.0; 95\% CI 1.0-4.0; $p=0.002$; Table 6). In-lesion angiographic binary restenosis was significantly higher in group CRYO (58.5\% vs. $22.7 \%, 74.1 \%$ vs. $47.0 \%$, and $100.0 \%$ vs. $78.9 \%$ $[p<0.006$ by log-rank test] at 12,24 , and 36 months for groups CRYO and COBA, respectively) (HR 1.3, 95\% CI $0.6-2.6[p=0.4$ by Cox model]; Table 5 and Fig. 3A). Binary in-lesion restenosis was also adversely affected by impaired renal function (HR 2.5, 95\% CI 1.4-4.6, $p=0.002$; Table 6; Fig. 3B). Subgroup bivariable Kaplan-Meier analysis evaluating outcomes of ISR lesions failed to identify any statistically significant difference between the two groups with regard to primary patency $(62.5 \%$ in group CRYO vs. $53.8 \%$ in group COBA, $p=0.97$ ), restenosis (100\% in group CRYO vs. $92.3 \%$ in group COBA, $p=0.71$ ), and TLR-free survival ( 41.6 vs. $45 \%$ in groups CRYO and COBA, respectively, $p=0.2$ ) at $\leq 36$ months.

In addition, significantly more TLR events due to recurrent symptoms were noted in group CRYO at $\leq 36$ months ( 48.4 vs. $26.5 \%, 66.5$ vs. $40.4 \%$, and 66.5 vs. $52.3 \%$ [ $p<0.04$ by log-rank test] at 12,24 , and 36 months, respectively). Cryoplasty also proved to be a highly significant predictor of increased TLR events (HR 2.5, 95\% CI $1.2-5.3, p=0.01$ by Cox model; Table 5; Fig. 4). Smoking, renal disease, IDDM, and hyperlipidemia were also calculated as predictive factors of increased clinically driven repeat procedures (Table 6).

\section{Discussion}

More than 200 million people worldwide have DM, $90 \%$ of which is insulin-resistant type II DM [25]. It is also well known that diabetic patients demonstrate a higher prevalence of PAOD. A large pool of cardiological studies have reported a significantly higher prevalence of coronary artery disease in diabetic compared with nondiabetic patients [26]. Notably, small-vessel coronary artery disease in diabetic patients is characterized by a more diffuse distribution and calcified pattern, numerous repeat procedures of revascularization owing to increased vascular restenosis, increased rates of major adverse cardiac events, and decreased survival [26-28]. With regard to the lower extremities, diabetic patients with intermittent claudication symptoms have an increased risk of acute limb ischemia, which also entails a higher major amputation risk, 35 and $21 \%$, respectively, according to one observational study, compared with 19 and $3 \%$ in nondiabetic patients, correspondingly [23].

Table 5 Bivariable and multivariable Cox proportional hazards regression analysis of primary end points stratified for CRYO versus COBA

\begin{tabular}{lclllll}
\hline Variable & CRYO $(\%)$ & COBA $(\%)$ & $p^{*}$ & HR $^{\dagger}$ & 95\% CI & Adjusted $p^{\dagger}$ \\
\hline Lesion primary patency & 59.2 & 54.6 & 0.894 & 2.2 & $1.1-4.3$ & 0.02 \\
Lesion binary restenosis & 100.0 & 78.9 & 0.006 & 1.3 & $0.6-2.6$ & 0.4 \\
TLR-free survival & 33.5 & 47.7 & 0.04 & 2.5 & $1.2-5.3$ & 0.01 \\
\hline
\end{tabular}

* Calculated by log-rank bivariable analysis

${ }^{\dagger}$ Cox model adjusted for IDDM, renal disease, smoking, hyperlipidemia, lesion grade, lesion type (de novo or ISR), heavy calcifications, TASC classification, and type of treatment (cryoplasty or COBA) 
Fig. 2 Primary patency stratified for cryoplasty and COBA. A Kaplan-Meier analysis plots and $\mathbf{B}$ Coxadjusted plots stratified for the type of treatment modality used (CRYO vs. COBA)

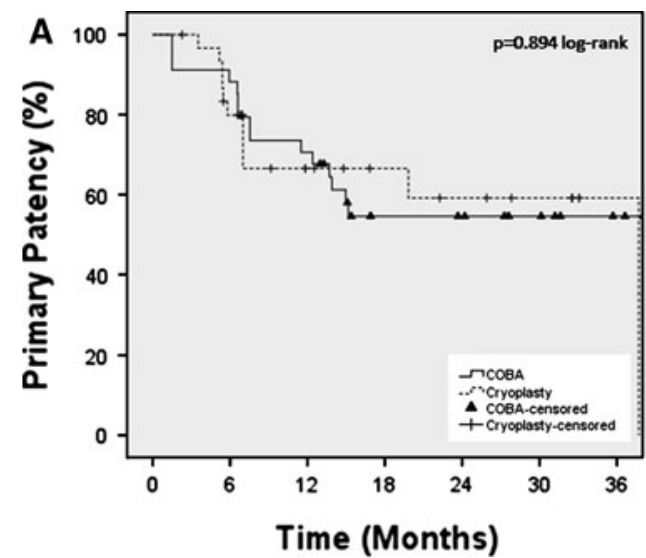

Table 6 Significant predictors of outcomes other than type of treatment as identified by the Cox model

\begin{tabular}{llccccc}
\hline Variable & Independent variable & Regression coefficient $\beta$ & SE & HR & 95\% CI & Adjusted $p$ \\
\hline Lesion primary patency & De novo lesions & 0.695 & 0.352 & 2.0 & $1.0-4.0$ & 0.04 \\
Lesion binary restenosis & Renal disease & 0.923 & 0.303 & 2.5 & $1.4-4.6$ & 0.002 \\
TLR-free survival & Smoking & 0.879 & 0.435 & 2.4 & $1.0-5.7$ & 0.04 \\
TLR-free survival & IDDM & -0.835 & 0.400 & 0.4 & $0.2-0.8$ & 0.03 \\
TLR-free survival & Renal disease & 0.984 & 0.407 & 2.7 & $1.2-5.9$ & 0.01 \\
TLR-free survival & Hyperlipidemia & -0.908 & 0.379 & 0.4 & $0.2-0.8$ & 0.01 \\
\hline
\end{tabular}

Cox model adjusted for IDDM, renal disease, smoking, hyperlipidemia, lesion grade, lesion type (de novo or ISR), heavy calcifications, and TASC and Rutherford classifications

$T L R$ target lesion revascularization, $H R$ hazard ratio, IDDM insulin-dependent diabetes mellitus
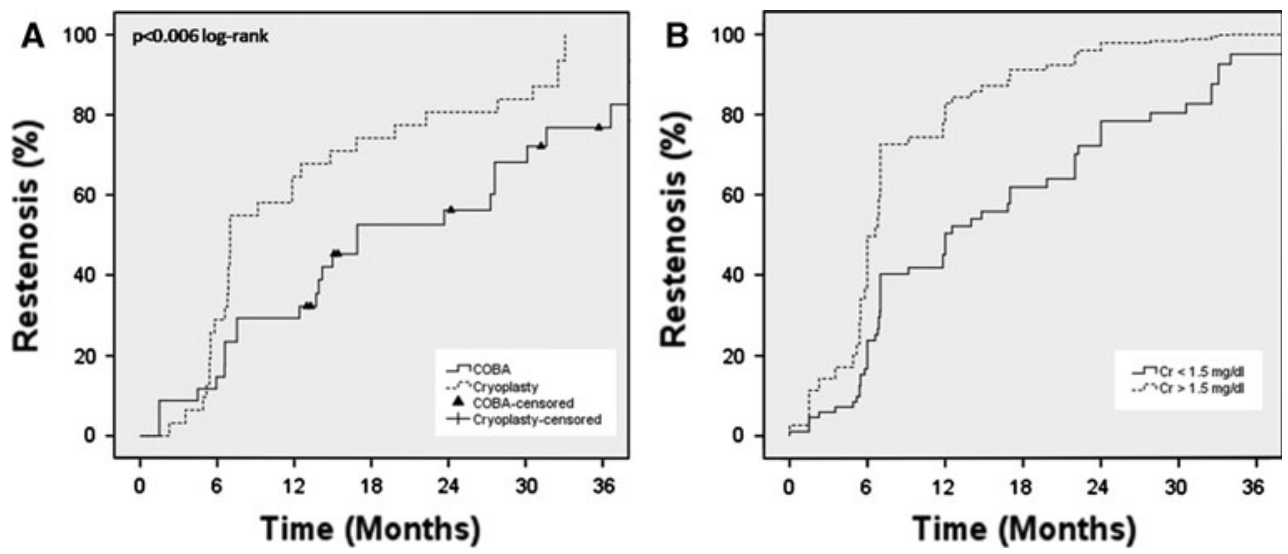

Fig. 3 Angiographic binary restenosis plots. A Kaplan-Meier curves of cryoplasty and COBA and B Cox adjusted plots stratified for renal disease (initial serum creatinine level $>1.5 \mathrm{mg} / \mathrm{dl}$ )

The aggressive nature of peripheral arterial disease in the setting of diabetes, by means of faster progression and wider lesion dissemination of the disease afflicting both the macrovasculature and microvasculature of the lower limbs, coupled with the rapidly increasing size of the affected population, has turned the interest of the research community toward novel therapies of revascularization $[9,11$, 29]. Atherosclerosis in diabetes exhibits a phenotypically different and more aggressive behavior than in normoglycemic patients. Diabetic vessels develop cylindrical calcifications of the media tunica, progressive intimal restenosis, and amplified rates of proliferation, adhesion, and migration of vascular smooth-muscle cells $[2,27,28]$. Patients with PAOD and diabetes experience walking difficulties and nonhealing foot ulcers not only because of inadequate blood supply to the limb and coexisting diabetic neuropathy but also due to alterations in normal muscle metabolism and glucose use [30-32]. The 
Fig. 4 TLR-free survival. A Kaplan-Meier analysis plots and $\mathbf{B}$ Cox-adjusted plots stratified CRYO versus COBA
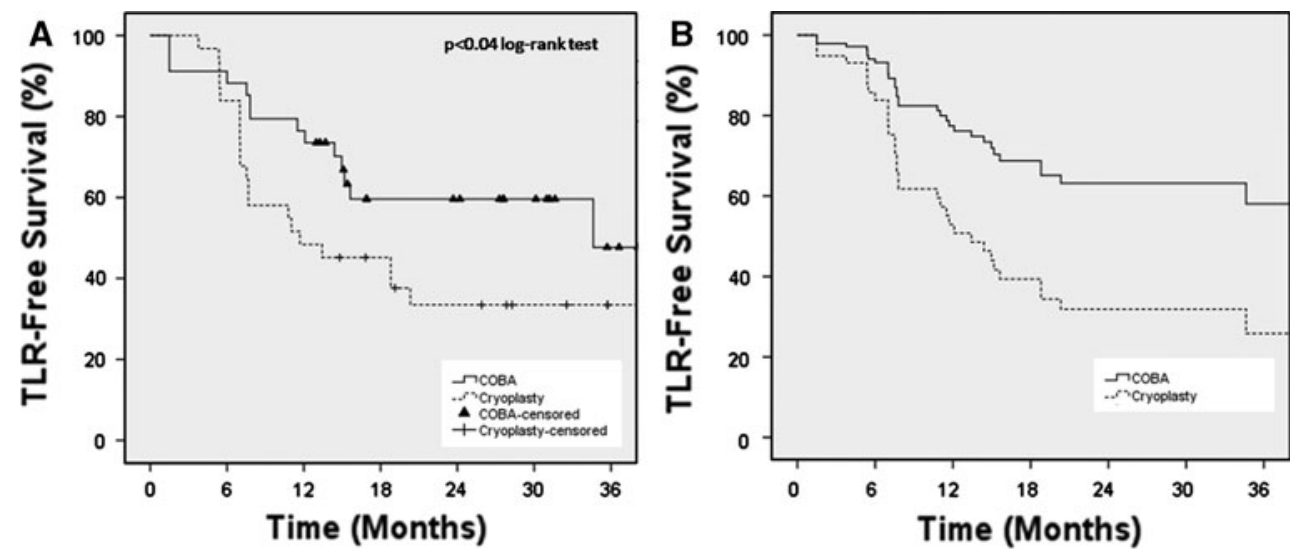

significant financial and social healthcare burden of diabetic vascular disease has prompted interventional specialists to apply aggressive risk-factor modification and extensive percutaneous recanalization procedures to prevent limb loss, increase vascular patency with less repeat procedures, and ultimately augment overall survival in this demanding patient group [9, 30].

The intense restenotic response due to neointimal hyperplasia and negative remodeling, which results in poor vascular patency after angioplasty and/or stenting of the SFA and/or PA of arteries in case of diabetes, has motivated us to study the potential therapeutic benefit of cryoplasty in these lesions. We conducted a prospective, single-center, randomized, controlled study comparing cryoplasty with standard balloon angioplasty. The initial hypothesis was that the presumed more uniform and less traumatic immediate performance of cryoplasty, in synergy with the induction of the apoptotic effect, would result in less neointimal hyperplasia and, as a consequence, better immediate and long-term angiographic and clinical results. Apart from a few uncontrolled cohort studies or case series, scientific data about the actual value of cryoplasty in terms of immediate technical success and late outcomes are scarce[16, 17]. To our knowledge, this is the first prospective randomized trial investigating the merits of this relatively new procedure in the demanding setting of diabetic femoropopliteal occlusive disease.

According to our results, cryoplasty was a safe procedure when applied in the femoropopliteal artery of diabetic patients and performed equally well compared with conventional balloon angioplasty with regard to immediate technical success (58.0 vs. $64.7 \%, p=0.4$ ) and long-term limb salvage ( 95.8 vs. $92.1 \%, p=0.54)$. However, the tested hypothesis, i.e., that cryoplasty would result in superior immediate technical success rates with less stent use due to flow-limiting dissection and/or elastic recoil, was not verified. In our study, application of cryoplasty showed only a nonsignificant trend toward less bail-out stenting $(25.8 \%$ in group CRYO vs. $35.3 \%$ in group COBA). Of note, nonetheless, despite the almost equivalent length of treated lesions and the number of stents used, there was a nonsignificant trend toward less overall stent length in group CRYO compared with control group COBA. Hence, one could argue that the notion of cryoplasty achieving more uniform plaque remodeling with less vessel wall barotrauma was neither confirmed nor actually rejected in this controlled trial. Literature reports about adjunctive bail-out stenting after cryoplasty are variable and range from $8.9 \%$ up to $33.3 \%$ in case of treatment of occluded lesions [16].

In a significant number of lesions that were particularly difficult to treat (20 of 31 [64.5\%]), probably because of the heavily calcified vessel wall, two consecutive cryoplasty applications were needed. This underscores the increased difficulty that heavily calcified diabetic lesions present to cryoplasty treatment. We speculate that the lower pressure ( $\leq 8 \mathrm{Atm})$ and the compliant nature of the cryoplasty catheter, which can be attributed to the compressible gas used for the inflation, compared with the uniformly liquid-inflated noncompliant or semicompliant conventional balloons, may result in more immediate technical failures, especially in the setting of resistant, eccentric, fibrotic, or fibrocalcific lesions. It should be stressed that although many operators may anecdotally perform multiple repeated cryoplasty sessions in the same lesion, i.e., during the same procedure, because of an initial suboptimal angioplasty result or in case of tandem lesions, there are no published data about the actual biomechanical effects as well as the induction of apoptosis after consecutive applications of freezing-thawing to the vessel wall [33, 34].

The Cox model identified a highly significant 2.2-fold increased hazard for reocclusion when cryoplasty was applied. Likewise, cryoplasty was a strong independent predictive factor associated with 2.5 times more events of repeat procedures of revascularization (TLR) in both bivariable and multivariable analysis. Moreover, the binary angiographic restenosis rate was significantly higher in the cryoplasty group, but this result was not confirmed by the adjusted $p$ value of the Cox model, which indicated 
impaired renal function as the only independent factor affecting restenosis. Arguably, however, the results reported herein are only applicable to the specific long, heavily calcified lesions of diabetic patients. In vivo experimental studies have also reported that cryoplasty induces probably more necrosis and inflammation, rather than apoptosis of smooth muscle cells, which are followed by a late aggressive neointimal hyperplastic response and vasoconstrictive wall remodeling [21, 35]. Of further interest, in vitro evaluation of cryoplasty in a calcified arterial wall with finite-element heat and mass-transfer computational models showed that temperatures as low as $-110^{\circ} \mathrm{C}$ may be necessary for effective cryothermal plaque ablation, which obviously is not feasible in the setting of a real clinical scenario [36].

Long-term primary patency probability rates of almost $60 \%$ in both groups (59\% in group CRYO vs. $55 \%$ in group COBA at 3 years) are similar to previously published data of endovascular SFA treatment $[10,11,13,14,29]$. We believe that these results are in line with the fact that the majority of the included patients suffered from severe intermittent claudication as well as high TASC grade and increased calcification of the treated lesions [10, 11, 37]. Furthermore, multivariable analysis with the Cox model isolated de novo atheromatous lesions, with almost two times higher risk of reocclusion, as a strong independent predictor of decreased vascular primary patency. Because the performance of cryoplasty was superior for ISR than for de novo lesions, this could encourage further investigations for the use of this technology in this particular group of revascularization procedures.

Recently published mid-term results from another prospective, single-center, randomized, controlled trial evaluating cryoplasty in the PA were similar to those reported herein. The investigators randomized 86 patients with atherosclerotic stenoses or occlusions of the PA between cryoplasty and conventional angioplasty treatment. Despite a nonsignificant trend toward higher 9-month primary patency (79\% after CRYO vs. $67 \%$ after COBA), cryoplasty posed comparable initial grade $\mathrm{C}$ dissections and adjunctive stent placement with significantly inferior immediate technical success ( 35 vs. $54 \%$ for conventional angioplasty) [38]. In line with the previously mentioned data, the pioneering investigators of cryoplasty critically reappraised their initial results in the femoropopliteal arteries because of poor long-term freedom of restenosis, which decreased to $<50 \%$ at 24 months. In a recent report, they explicitly stated that considering its additional costs, they had abandoned use of cryoplasty in their everyday practice $[39,40]$.

In contrast, 12-month follow-up results from the BTK Chill Trial, which evaluated cryoplasty in the infrapopliteal arteries of CLI patients, reported high rates of acute technical success and major amputation-free interval (97.3\% and $78.5 \%$, respectively) followed by a low percentage of clinically driven repeat procedures $(21 \%$ at 12 months) [41]. Almost $70 \%$ of the patients had diabetes. The above-mentioned differences in anatomic success and TLR-free interval rates between femoropopliteal and infrapopliteal cryoplasty trials could be accredited to smaller vessel diameter and different biomechanics of the belowthe-knee anatomic region compared with the femoropopliteal artery.

Study limitations include the relatively small number of patients assigned to each group (thus underpowering the study for the extraction of more statistically significant results and better stratification of risk factors between the two groups) as well as the inherent bias of the single-center nature of the study. We should also underscore the off-label use of cryoplasty in heavily calcified lesions (approximately $30 \%$ of the total lesions treated in both groups). Of note, only $41.4 \%$ of patients in group CRYO and $38.7 \%$ in group COBA had CLI, and only a small percentage of the lesions in both groups were chronic total occlusions. As a result, patients included in the study might not be the most representative cases of a diabetic population. However, the study's main intention was to compare the efficacy of cryoplasty and COBA as an SFA recanalization tool with regard to primary patency, restenosis, and TLR-free survival rates. Nonetheless, according to our results and until further scientific evidence becomes available, the use of cryoplasty should be limited to selected angioplasty cases within properly designed clinical studies. We have shown that cryoplasty, in this particular group of diabetic patients, is a safe but not more efficient method of endovascular treatment than COBA. No substantial benefit was shown in terms of immediate technical success, stent use, and longterm primary patency. Furthermore, the restenosis and target lesion revascularization rates were higher, additionally compromising the cost-effectiveness of the procedure taking into account the higher cost of the PolarCath balloon and the comparable rates of adjunctive stent use. To conclude, cryoplasty of the femoropopliteal artery in diabetic patients was associated with lower primary patency and more clinically driven repeat procedures after long-term follow-up compared with conventional balloon angioplasty.

Conflict of interest statement The authors declare that they have no conflict of interest.

\section{References}

1. King H, Aubert RE, Herman WH (1998) Global burden of diabetes, 1995-2025: prevalence, numerical estimates, and projections. Diabetes Care 21:1414-1431 
2. Faries PL, Rohan DI, Takahara H et al (2001) Human vascular smooth muscle cells of diabetic origin exhibit increased proliferation, adhesion, and migration. J Vasc Surg 33:601-607

3. Melliere D, Berrahal D, Desgranges P et al (1999) Influence of diabetes on revascularisation procedures of the aorta and lower limb arteries: early results. Eur J Vasc Endovasc Surg 17:438-441

4. Siablis D, Karnabatidis D, Katsanos K et al (2007) Infrapopliteal application of paclitaxel-eluting stents for critical limb ischemia: midterm angiographic and clinical results. J Vasc Interv Radiol 18:1351-1361

5. DeRubertis BG, Faries PL, McKinsey JF et al (2007) Shifting paradigms in the treatment of lower extremity vascular disease: a report of 1000 percutaneous interventions. Ann Surg 246:415422; discussion 422-424

6. DeRubertis BG, Pierce M, Ryer EJ et al (2008) Reduced primary patency rate in diabetic patients after percutaneous intervention results from more frequent presentation with limb-threatening ischemia. J Vasc Surg 47:101-108

7. Rutherford RB, Jones DN, Bergentz SE et al (1988) Factors affecting the patency of infrainguinal bypass. J Vasc Surg 8:236246

8. Maca T, Schillinger M, Hamwi A et al (2005) Insulin, C-peptide, and restenosis after femoral artery balloon angioplasty in type II diabetic and nondiabetic patients. J Vasc Interv Radiol 16:31-35

9. Clark TW, Groffsky JL, Soulen MC (2001) Predictors of longterm patency after femoropopliteal angioplasty: results from the STAR registry. J Vasc Interv Radiol 12:923-933

10. Muradin GS, Bosch JL, Stijnen T et al (2001) Balloon dilation and stent implantation for treatment of femoropopliteal arterial disease: meta-analysis. Radiology 221:137-145

11. Schillinger M, Sabeti S, Loewe C et al (2006) Balloon angioplasty versus implantation of nitinol stents in the superficial femoral artery. N Engl J Med 354:1879-1888

12. Laird JR, Biamino G, McNamara T et al (2006) Cryoplasty for the treatment of femoropopliteal arterial disease: extended follow-up results. J Endovasc Ther 13(Suppl 2):II52-II59

13. Zabakis P, Kardamakis DM, Siablis D et al (2005) External beam radiation therapy reduces the rate of re-stenosis in patients treated with femoral stenting: results of a randomised study. Radiother Oncol 74:11-16

14. Rogers JH, Laird JR (2007) Overview of new technologies for lower extremity revascularization. Circulation 116:2072-2085

15. Bakken AM, Palchik E, Hart JP et al (2007) Impact of diabetes mellitus on outcomes of superficial femoral artery endoluminal interventions. J Vasc Surg 46:946-958

16. Wildgruber MG, Berger HJ (2008) Cryoplasty for the prevention of arterial restenosis. Cardiovasc Intervent Radiol 31:1050-1058

17. McCaslin JE, Macdonald S, Stansby G (2007) Cryoplasty for peripheral vascular disease. Cochrane Database Syst Rev (4):CD005507

18. Thomsen HS (2006) European Society of Urogenital Radiology (ESUR) guidelines on the safe use of iodinated contrast media. Eur J Radiol 60:307-313

19. Bennett MR (1999) Apoptosis of vascular smooth muscle cells in vascular remodelling and atherosclerotic plaque rupture. Cardiovasc Res 41:361-368

20. Tatsutani KN, Joye JD, Virmani R et al (2005) In vitro evaluation of vascular endothelial and smooth muscle cell survival and apoptosis in response to hypothermia and freezing. Cryo Lett 26:55-64

21. Grassl ED, Bischof JC (2005) In vitro model systems for evaluation of smooth muscle cell response to cryoplasty. Cryobiology 50:162-173

22. Sacks D, Marinelli DL, Martin LG et al (2003) Reporting standards for clinical evaluation of new peripheral arterial revascularization devices. J Vasc Interv Radiol 14(9 Pt 2):S395-S404
23. Norgren L, Hiatt WR, Dormandy JA et al (2007) Inter-Society Consensus for the Management of Peripheral Arterial Disease (TASC II). Eur J Vasc Endovasc Surg 33(Suppl 1):S1-S75

24. Siablis D, Kraniotis P, Karnabatidis D et al (2005) Sirolimuseluting versus bare stents for bailout after suboptimal infrapopliteal angioplasty for critical limb ischemia: 6-month angiographic results from a nonrandomized prospective single-center study. J Endovasc Ther 12:685-695

25. Nguyen LL (2007) Percutaneous treatment of peripheral vascular disease: the role of diabetes and inflammation. J Vasc Surg 45(Suppl A):A149-A157

26. Bax JJ, Inzucchi SE, Bonow RO et al (2007) Cardiac imaging for risk stratification in diabetes. Diabetes Care 30:1295-1304

27. Gilbert J, Raboud J, Zinman B (2004) Meta-analysis of the effect of diabetes on restenosis rates among patients receiving coronary angioplasty stenting. Diabetes Care 27:990-994

28. Mehran R, Dangas GD, Kobayashi Y et al (2004) Short- and long-term results after multivessel stenting in diabetic patients. $\mathrm{J}$ Am Coll Cardiol 43:1348-1354

29. Sabeti S, Schillinger M, Amighi J et al (2004) Primary patency of femoropopliteal arteries treated with nitinol versus stainless steel self-expanding stents: propensity score-adjusted analysis. Radiology 232:516-521

30. Stratton IM, Adler AI, Neil HA et al (2000) Association of glycaemia with macrovascular and microvascular complications of type 2 diabetes (UKPDS 35): prospective observational study. Br Med J 321(7258):405-412

31. Barker GA, Green S, Walker PJ (2004) Effect of carbohydrate supplementation on walking performance in peripheral arterial disease: a preliminary physiologic study. J Vasc Surg 40:932-938

32. Dolan NC, Liu K, Criqui MH et al (2002) Peripheral artery disease, diabetes, and reduced lower extremity functioning. Diabetes Care 25:113-120

33. Korteweg MA, van Gils M, Hoedt MT et al (2009) Cryoplasty for occlusive disease of the femoropopliteal arteries: 1-year followup. Cardiovasc Intervent Radiol 32:221-225

34. Venkatasubramanian RT, Wolkers WF, Shenoi MM et al (2010) Freeze-thaw induced biomechanical changes in arteries: role of collagen matrix and smooth muscle cells. Ann Biomed Eng 38:694-706

35. Balasubramanian SK, Venkatasubramanian RT, Menon A et al (2008) Thermal injury prediction during cryoplasty through in vitro characterization of smooth muscle cell biophysics and viability. Ann Biomed Eng 36:86-101

36. Men-Chi H, Ravigururajan TS (2007) Biothermal modeling of post-cryoplasty atherosclerosis in restenotic patients. Cardiovasc Eng 7:7-16

37. Schillinger M, Sabeti S, Dick P et al (2007) Sustained benefit at 2 years of primary femoropopliteal stenting compared with balloon angioplasty with optional stenting. Circulation 115:27452749

38. Jahnke T, Mueller-Huelsbeck S, Charalambous N et al (2010) Prospective, randomized single-center trial to compare cryoplasty versus conventional angioplasty in the popliteal artery: midterm results of the COLD study. J Vasc Interv Radiol 21:186-194

39. Samson RH, Showalter DP, Lepore M Jr et al (2008) Cryoplasty therapy of the superficial femoral and popliteal arteries: a reappraisal after 44 months' experience. J Vasc Surg 48:634-637

40. Samson RH, Showalter DP, Lepore MR Jr et al (2006) Cryoplasty therapy of the superficial femoral and popliteal arteries: a single center experience. Vasc Endovascular Surg 40:446-450

41. Das TS, McNamara T, Gray B et al (2009) Primary cryoplasty therapy provides durable support for limb salvage in critical limb ischemia patients with infrapopliteal lesions: 12-month follow-up results from the BTK Chill Trial. J Endovasc Ther 16(Suppl 2):III9-II30 